ORIGINAL ARTICLE

AFRICAN JOURNAL OF CLINICAL AND EXPERIMENTAL MICROBIOLOGY

AJCEM/201002/21023

COPYRIGHT 2010

AFR. J. CLN. EXPER. MICROBIOL 11(3): 174-178
SEPTMBER 2010 ISBN 1595-689X VOL 11(3)

-http://www.ajol.info/journals/ajcem

\title{
THE EFFECTS OF ANTIRETROVIRAL TREATMENT ON LIVER FUNCTION ENZYMES AMONG HIV-INFECTED OUT PATIENTS ATTENDING THE CENTRAL HOSPITAL OF YAOUNDÉ, CAMEROON
}

Lucien $^{1 *}$, K. F. H. , Clement ${ }^{1}$, A,N.J., Fon ${ }^{1}$, N. P., Weledji ${ }^{1}$ P. and Ndikvu, ${ }^{2}$ C. P. ${ }^{1}$ Faculty of Health Sciences, University of Buea, Republic of Cameroon and ${ }^{2}$ Faculty of Science, University of Buea, Republic of Cameroon

* Correspondence: Kamga Fouamno Henri Lucien (PhD), Faculty of Health Sciences, University of Buea, P.O Box, 63, Buea, Republic of Cameroon, Phone number (+237) 99721972. E-mail: henrikamga2002@yahoo.fr

\begin{abstract}
The emergence of liver diseases as one of the major causes of death in people infected with HIV has paralleled the introduction of more effective antiretroviral therapies. This study was carried out with the aim of determining the effects of antiretroviral treatment on liver enzymes (SGOT and SGPT) in patients placed on antiretroviral therapy. A prospective cross-sectional 3 years study was carried out among patients confirmed to be HIV positive and who were to be placed on antiretroviral drugs at the HIV/AIDS out patient clinic of the Yaoundé Central Hospital, . Cameroon. Levels of transaminases of patients were measured in four phases using the International Federation of Clinical Chemistry (IFCC) protocol. Of the 150 patients who participated in the study, $54.0 \%$ (81/150) presented with transaminitis at the final phase of the study with respect to aspartate aminotransferase (AST), 77.78\% (63/81) of whose AST levels only increased after initiation of highly active antiretroviral therapy (HAART). $22.67 \%(34 / 150)$ presented with transaminitis with respect to alanine aminotransferase (ALT). At the final phase, $70.58 \%$ of whose ALT levels only increased after HAART initiation. Increase in blood transaminase levels was statistically independent on age group and the drug combinations. Increase in AST levels was associated with an increase in ALT levels upon treatment $(r=0.58)$. There was a significant positive linear relationship between duration of treatment and concentration of transaminases over the years $(r=0.9)$. We therefore concluded that highly active antiretroviral therapy (HAART) is associated with low level hepatotoxicity at therapy initiation, regardless of drug class or combination.
\end{abstract}

Keywords: Antiretroviral treatment, Liver function enzymes, Hepatotoxicity, Highly active antiretroviral therapy

\section{Introduction}

Antiretroviral drug-related liver injury (ARLI) is defined by elevations in liver enzymes in serum, with alanine aminotransferase (ALT) characteristically greater than aspartate aminotransferase (AST). It is one of the greatest causes of treatment discontinuation in HIV-infected patients [1]. Its prevention and management is therefore very important among HIV-infected patients who are to be placed on highly active antiretroviral therapy (HAART) [2]. Till date, there has been broad variability in the criteria used in clinical studies to categorize the severity of hepatotoxicity. Some studies have utilized ALT parameters as minimal as two times the upper limits of normal [3] while others have employed an absolute threshold (e.g., >100 IU/ml), regardless of baseline liver function tests [4]. Surveys have reported an increased incidence of hepatic injury in HAART-treated patients and identified lifethreatening hepatotoxic events and end-stage liver disease in patients on antiretroviral [5].
With the widespread use of HAART and the availability of new antiretroviral medications, ARLI has gained prominent attention owing to its negative impact on clinical outcomes. Drugassociated hepatotoxicity also creates an economic burden on already strained medical budgets, since additional visits and hospital admissions are often required for appropriate patient care and management [1]. Furthermore, antiretroviral drug discontinuation hampers maintenance of HIV suppression. The severity of ARLI may range from the absence of symptoms to liver decompensation, and the outcome can range from spontaneous resolution to liver failure and death [6].

In August 2003, Cameroon defined and included first and second line drug regimens in the national treatment guidelines, including different HIV-drug combinations based on $\mathrm{WHO}$ recommendations and in 2005, the number of people living with HIV was estimated at 505.000 , among which $61 \%$ were 
females. An estimated number of 108,000 Cameroonians (0-49 years) were reported to be in need of antiretroviral therapy. This figure has been on the rise given that the number of people infected with the virus increased on daily basis and subsequently required treatment [7].

Several highly active antiretroviral therapy (HAART) regimens are hepatotoxic and the liver is one of the vital organs useful in the metabolism of these drugs as well as in detoxification. It is therefore important that the liver which is the main biochemical hub of the body be monitored and those HAART regimens that may be toxic to it identified so that changes or modifications can be made to enhance patient care.

\section{Materials and Methods Study Design}

A cross-sectional study that involved newly diagnosed HIV positive individuals who reported for treatment at the outpatient clinic of the Yaounde central Hospital in Cameroon was done. Information extraction forms were used to collect data from patients' files.

\section{Study area}

Yaoundé is the administrative capital of Cameroon with a population of 143000 inhabitants. It is centrally located and made up of immigrants from all the regions of Cameroon. The outpatient clinic of the Yaoundé Central Hospital is in the heart of the city.

\section{Study population}

The study was carried out on volunteer patients already diagnosed and confirmed to be HIV positive. Patients were made to understand that it was not a hospital obligation for them to participate in the research and neither was it a pre-requisite to accessing routine medical services. Written informed consent forms were distributed and explained to the participants one week prior to the beginning of the samples collection. Only HIV positive volunteers who returned their informed consent forms duly signed were recruited irrespective of their gender or clinical state of the disease. Only participants who were 20 years and above were recruited in the study

\section{Phases of the study}

The study was conducted for a period of three (3) years, from November 2006 to December 2009. Blood samples were collected in four (4) phases as follows.

- $\quad$ Phase 1: in November 2006, before patients were placed on antiretroviral therapy

- Phase 2: in November 2007 one year after commencement of therapy

- $\quad$ Phase 3: in November 2008, two years after commencement of therapy

- $\quad$ Phase 4: December 2009, final phase.

\section{Laboratory procedure}

About $4 \mathrm{ml}$ of blood samples were collected by venipuncture into labelled (coded) dry test tubes. After collection, samples were irreversibly de-linked from donor's information except that concerning the age. Blood samples were allowed to coagulate after which they were centrifuged at $3000 \mathrm{rpm}$ for 5 minutes to obtain sera.

\section{Measurement of ALT (SGPT)}

Measurement of ALT was done following a method that has been made according to the 2002 International Federation of Clinical Chemistry (IFCC) protocol [8]. Commercial kits produced by HOSPITEX DIAGNOSTICS Ltd were used. The test was carried out using the monoreagent procedure.

The working reagent was prepared by mixing 4 volumes of reagent one (R1) in 1 volume of reagent two (R2). Mixed by inversion and stored away from any light sources at $2-8^{\circ} \mathrm{C}$.

The spectrophotometer was calibrated for ALT measurement. A series of labelled test tubes, i.e. Blank, normal control and patients from $1,2 \ldots \mathrm{n}^{\text {th }}$ according to the number of samples to be analysed.

\begin{tabular}{|l|l|l|}
\hline & $\begin{array}{l}\text { Working } \\
\text { reagent }\end{array}$ & Sample \\
\hline Blank & $1 \mathrm{ml}(1000 \mathrm{ul})$ & \\
\hline $\begin{array}{l}\text { Normal } \\
\text { control }\end{array}$ & $1 \mathrm{ml}$ & $100 \mathrm{ul}$ \\
\hline Patient & $1 \mathrm{ml}$ & $100 \mathrm{ul}$ \\
\hline
\end{tabular}

The preparation was mixed and the first reading of absorbance was executed after 90 seconds. Incubating at $37^{\circ} \mathrm{C}, 3$ other readings were performed at 60 seconds interval. The change in absorbance per minute was then calculated.

The activities were obtained from the following calculations:

340nm: Activity $(\mathrm{U} / \mathrm{L})$ = change in absorbance / min. $x 1769$.

Normal values for ALT (SGPT) at $37^{\circ} \mathrm{C}$ : Women up to $34 \mathrm{U} / \mathrm{L}$. Men up to $45 \mathrm{U} / \mathrm{L}$.

\section{Measurement of AST (SGOT)}

Measurement of AST was done following a method that has been made according to the 2002 International Federation of Clinical Chemistry (IFCC) protocol [8]. Commercial kits produced by HOSPITEX DIAGNOSTICS Ltd were used. The test was conducted using the monoreagent procedure.

The working reagent was prepared by adding the vial of reagent two (R2) in the vial of reagent one (R1). The reagent was then stored at $2-8^{\circ} \mathrm{C}$ away from any light sources.

The spectrophotometer was calibrated for AST measurement. A series of labelled test tubes, i.e. Blank, normal control and patients from $1,2 \ldots . n^{\text {th }}$ according to the number of samples to be analysed. The spectrophotometer was brought to zero against distilled water. 


\begin{tabular}{|l|l|l|}
\hline & $\begin{array}{l}\text { Working } \\
\text { reagent }\end{array}$ & Sample \\
\hline Blank & $1 \mathrm{ml}(1000 \mathrm{ul})$ & \\
\hline $\begin{array}{l}\text { Normal } \\
\text { control }\end{array}$ & $1 \mathrm{ml}$ & $100 \mathrm{ul}$ \\
\hline Patient & $1 \mathrm{ml}$ & $100 \mathrm{ul}$ \\
\hline
\end{tabular}

The preparation was mixed and the first reading of absorbance was executed after 90 seconds. Incubating at $37^{\circ} \mathrm{C}, 3$ other readings were performed at 60 seconds interval. The change in absorbance per minute was then calculated as follows:

340nm: Activity (U/L) = Change in absorbance/ minute $x 1769$.

(Normal values for AST (SGOT): Women up to 31U/L and Men up to 35U/L).

\section{Statistical Analysis}

Statistical tests such as Chi-square and Pearson's product moment correlation were used to test for the significance of the results obtained.

\section{Results}

Of the 150 patients who participated in this study, 81 $(54.0 \%)$ showed a transaminitis with respect to AST at the final phase of the study, $77.78 \%(63 / 81)$ of whose AST levels only increased after initiation of HAART. $22.67 \% \quad(34 / 150)$ presented with transaminitis with respect to ALT at the final phase, $70.58 \%$ of whose ALT levels only increased at HAART initiation. Increase in AST levels was found to be is associated with an increase in ALT levels upon treatment $(\mathrm{r}=0.58)$.

Figure 1 and Figure 2 show the relationship between the mean concentration of transaminases (SGOT and SGPT) and the duration of treatment. There was a linear increase of SGOT mean concentration in blood over time $(\mathrm{r}=0.81)$.

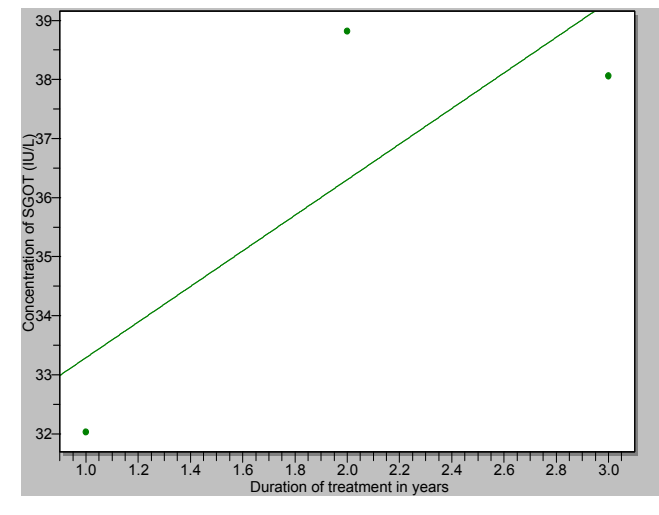

$(\mathrm{r}=0.81)$
Figure 1: The relationship between the mean concentration of SGOT and duration of treatment

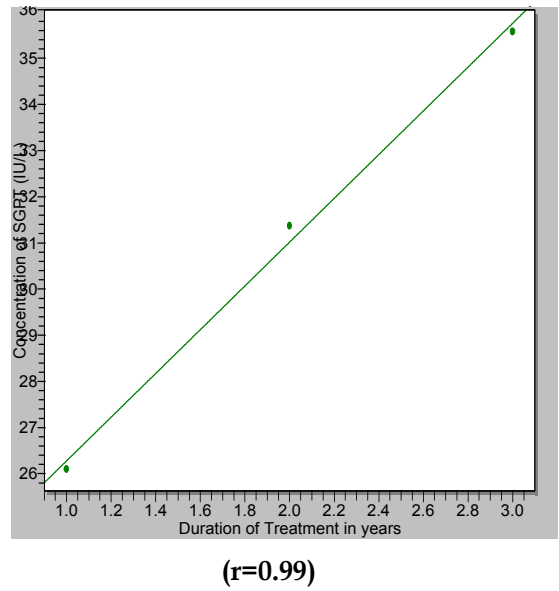

Figure 2: The relationship between the mean concentration of SGPT and duration of treatment

Also, there was a significant positive linear relationship between the concentration of transaminase (SGPT) with treatment duration $(\mathrm{r}=0.99)$. The level of transaminases concentration was found to be increasing over time.

Tables 1 shows the age groups and AST levels among the 150 patients. The both transaminase levels were highest among the patients aged above 50 years.

Table 2 shows the drug combinations and transaminase (AST and ALT) levels. Stocrin,Duovir was the combination showing the highest percentage of increased AST (55.17\%) while LamivirS showed the highest percentage of increased ALT (35.29\%).

Table1: Age groups and transaminase (AST and ALT) levels

\begin{tabular}{|l|l|l|l|}
\hline $\begin{array}{l}\text { Age } \\
\text { group } \\
\text { (year) }\end{array}$ & Total & $\begin{array}{l}\text { Number(\%)* } \\
\text { showing } \\
\text { high AST } \\
\text { levels }\end{array}$ & $\begin{array}{l}\text { Number(\%) } \\
\text { showing } \\
\text { high ALT } \\
\text { levels }\end{array}$ \\
\hline $20-35$ & 73 & $32(21.33)$ & $19(26.02)$ \\
\hline $36-50$ & 57 & $29(50.87)$ & $13(22.80)$ \\
\hline$>50$ & 20 & $14(70)$ & $8(40)$ \\
\hline Total & 150 & $75(50)$ & $40(26.67)$ \\
\hline
\end{tabular}




\begin{tabular}{|l|l|l|l|}
\hline rug combination & Total & $\begin{array}{l}\text { Number(\%* showing } \\
\text { high AST levels }\end{array}$ & $\begin{array}{l}\text { Number(\%)* } \\
\text { showing high ALT levels }\end{array}$ \\
\hline ZidolamN & 24 & $9(37.5)$ & $6(25)$ \\
\hline LamivirS & 17 & $7(41.18)$ & $6(35.29)$ \\
\hline Triomine & 51 & $33(64.7)$ & $15(29.41)$ \\
\hline $\begin{array}{l}\text { Stocrin, } \\
\text { Duovir }\end{array}$ & 58 & $32(55.17)$ & $7(12.07)$ \\
\hline Total & 150 & $81(54.0)$ & $34(22.67)$ \\
\hline
\end{tabular}

Table 2: Drug combinations and transaminase (AST and ALT) levels

*percentage based on number of participant using the same drug combination $X^{2}=5.78 \quad P>0.05$

\section{Discussion}

In 2004 Cameroon was still in the context of generalized HIV epidemic, with a prevalence of 5,5\% of which $6,8 \%$ were women and $4,1 \%$ were men. Groups identified to have high risk behaviours included mostly men and women in uniform, commercial sex workers, truck drivers and populations living along the Chad - Cameroon pipeline project area. Youths around 20 years of age were found to be the most vulnerable. The major mode of transmission was through non - protected heterosexual activity, even if mother to child transmission remained a preoccupation [9], [8]. These are reasons why the study population sample included only individuals above.

In the present study, the level of transaminases concentration was found to be increasing over time. These results tie with those obtained in a previous retrospective review of more than 10,000 adults living with AIDS in Boston, where researchers confirmed that long time antiretroviral therapy is associated with high rate of severe hepatotoxicity regardless of drug class or combination [5].

In other studies, a different pattern of drug injury with nevirapine use has emerged, with onset of liver enzyme elevations occurring beyond 16 weeks of therapy, consistent with direct or idiosyncratic hostmediated liver injury [10]. Rates of hepatotoxicity from various registration trials were ranged from $1 \%$ to $9.5 \%$, but few patients have shown serious liverrelated outcomes [11]

Also, based on the WHO toxicity scale, the greatest percentage of patients who presented with elevated levels of transaminases $(76.81 \%$ for AST and $53.33 \%$ for ALT) were found to present with first degree hepatotoxicity which corresponds to low level liver toxicity. However, this does not tie with reports by spengler et al.[5] in which it was pointed out that longitudinal surveys have not only reported increased incidence of ARLI in patients but also identified life-threatening hepatotoxic events and end-stage liver disease in patients on antiretroviral therapy. The term hepatotoxicity may be misleading in the case of HIV drugs as some of the elevated liver enzymes may be due to other causes such as acute viral hepatitis, reactivation of chronic hepatitis B or C, alcohol ingestion as well as complementary drugs or medicines associated with HAART several of which have been noticed to be associated with clear-cut drug-induced hepatitis (DIH) for example used for the treatment of Tuberculosis [12]

We have found transaminase levels to be highest among the patients aged above 50 years. Although this finding was not statistically significant, it was in accordance with that of spengler et al. [5], but did not tie with a more recent report[13] in which younger diabetic patients had a higher tendency to have elevated ALT compared to those over 65 years. Our study shows significant relationship between the age of the patient and the level of transaminases concentration. Other studies have indicated that certain co morbidities, such as chronic hepatitis B (HBV) or hepatitis $\mathrm{C}(\mathrm{HCV})$ infection, may predispose patients to ARLI [10]. Highly active antiretroviral therapy (HAART) was found to be associated with low level hepatotoxicity at initiation, regardless of drug class or combination. Elevated transaminases due to antiretroviral therapy was independent on patient's age and increased levels of transaminases showed a significant positive linear relationship with increase in the duration of treatment. We therefore recommended that levels of transaminases should be followed up after initiation of antiretroviral therapy for all patients irrespective of their drug combination or age. The role of liver steatosis as a mechanism of, and as predisposing factor to anti retroviral drugrelated liver injury (ARLI) should be investigated. 


\section{REFERENCES}

1- Nuñez, M.J., Martin-Carbonero, L., Moreno, V., Valencia, E., Garcia-Samaniego, J., and Gonzalez-

Castillo, J. Impact of antiretroviral. AIDS 2006; 22: 825-829.

2- Palella, F., Baker, R., Moorman, A., Chmiel, J., Wood, K. and Brooks, J.(2006). Mortality in the highly active antiretroviral therapy era: changing causes of death and disease in the HIV outpatient study. Journal of Acquired Immune Deficiency Syndrome 2006; 43: 27-34.

3- Hernandez, L., Gilson, I., Jacobson, J., Affi, A., Puetz, T. and Dindzans, V. Antiretroviral hepatotoxicity in HIV-infected patients. Alimentary Pharmacology \& Therapeutics 2001; 15:1627-1632.

4- den Brinker, Marieke; Wit, Ferdinand W. N. M.; Wertheim-van Dillen, Pauline M. E.; Jurriaans, Suzanne; Weel, Jan; van Leeuwen, Remko; Pakker, Nadine G.; Reiss, Peter; Danner, Sven A.; Jan Weverling, Gerrit; Lange and Joep M. A. Hepatitis B and C virus co-infection and the risk for hepatotoxicity of highly active antiretroviral therapy in HIV- infection. AIDS 2000 14: 28952902

5- Spengler, U; Lichterfeld, M.; Rockstroh, J.K. Antiretroviral drug toxicity-Therapy. Reuters Health 2002

6- Clark, S., Creighton, S., Portmann, B., Taylor, C., Wendon, J. and Cramp M. Acute liver failure associated with antiretroviral treatment for HIV: a report of six cases. Journal of Hepatology 2002; 36:295-301.

7- Ministry of Public Heath. 2006-2010 National Strategic Plan for the fight against AIDS in Cameroun. Republic of Cameroon.

8- International Federation of Clinical Chemistry (IFCC). www.ifcc.org

9- Organisation des Nations Unies pour la lutte contre le Sida (ONUSIDA). Rapport annuel 2003. Genève: ONUSIDA

10- Soriano, V., Puoti, M., Garcia-Gascó, P., Rockstroh, J. K., Benhamou, Y., Barreiro, P. and McGovern, B. Antiretroviral drugs and liver injury AIDS 2001; 22: 1-13

11- Sulkowski, M., Thomas, D., Chaisson, R. and Moore, R. Hepatotoxicity associated with antiretroviral therapy in adults infected with HIV and the role of hepatitis C or B virus infection. Journal of the American Medeical Association 2004; 283:74-80.

12- Lewis, J. H. The rational use of potentially hepatotoxic medications in patients with underlying liver disease. Informa Healthcare 2002; 1:159-172

.13- Layla Judi, Ala Toukan, Yousef Khader, Kamel Ajlouni and $\mathrm{M}$ Amer Khatib. Prevalence of elevated hepatic transaminases among Jordanian patients with type 2 diabetes mellitus. Annals of Saudi Medicine 2010; 30: 25-32 